\title{
Inequalities of wealth distribution in a conservative economy
}

\author{
S. Pianegonda ${ }^{\text {a,* }}$, J. R. Iglesias ${ }^{\text {a }}$, \\ ${ }^{a}$ Instituto de Física, U.F.R.G.S., C.P. 15051, 91501-970 Porto Alegre - RS, \\ Brazil.
}

\begin{abstract}
We analyze a conservative market model for the competition among economic agents in a close society. A minimum dynamics ensures that the poorest agent has a chance to improve its economic welfare. After a transient, the system self-organizes into a critical state where the wealth distribution have a minimum threshold, with almost no agent below this poverty line, also, very few extremely rich agents are stable in time. Above the poverty line the distribution follows an exponential behavior. The local solution exhibits a low Gini index, while the mean field solution of the model generates a wealth distribution similar to welfare states like Sweden.
\end{abstract}

Key words: econophysics, inequalities, wealth distribution, minimum dynamics, PACS: 89.65.Gh, 89.75.Fb, 05.65.+b, 87.23.Ge

\section{Introduction}

The study of the distribution of the income of workers, companies and countries started a little more than a century ago when Italian economist Vilfredo Pareto investigated data of personal income for different European countries and found a power law distribution that seems not to depend on different economic conditions of the countries. In 1887, in his book Cours d'Economie Politique (1) he asserted that in all countries and times the distribution of

* Corresponding author Email address: salete@if .ufrgs.br (S. Pianegonda).

1 We acknowledge fruitful discussions with G. Abramson, R. Donangelo, S. Gonçalves, S. Risau-Gusman and J.L. Vega, and financial support from CNPq, Brazil

Preprint submitted to Elsevier Science 28 October 2018 
income and wealth follows a power law behavior where the cumulative probability $P(w)$ of people whose income is at least $w$ is given by $P(w) \propto w^{-\alpha}$, where the exponent $\alpha$ is named Pareto index. This universal power law is now called Pareto law and he found himself that the index assumes values typically close to 1.5 for several countries. The reduction of the slope $\alpha$ denotes a higher inequality of the income distribution.

However, recent data suggest that Pareto's distribution does not agree with observed data over the middle range of income, but it is generally accepted that it provides a good fit to the distribution of high range of income. Many other distributions of income were proposed, supported by international empirical data, and the distribution behaves in a log-normal, exponential or Levy way (2) in the middle income range. For instance, there are data from Japan and USA that are fitted by a log-normal distribution in middle range plus a power law for high income one (3), data from United Kingdom income and wealth fitted by an exponential law (middle range) and a power law (high range) (4) and data from Brazil (5) worker's income and enterprise capital that are fitted by a log-normal plus a power law for high range of income (6).

One of the goals of the study of wealth distribution is to determine the social inequalities implied in a given model of economic exchanges. Many ways to quantitatively measure the fairness of a given income distribution have been proposed in the economic science. Among them, the Gini index is well known and frequently used. In brief, for any given wealth density function $p(x)$, with mean $\rho$ and a given number $\nu \in[0,1], P(\leq \nu)=\int_{0}^{\nu} p(x) d x$ represents the cumulative fraction of population with wealth bellow $\nu$ and Lorenz function $L(\nu)$ is defined as the fraction of the total wealth which is attributed to the poorest $100 \nu$ percentage of the society. It easy to show that $L(\nu)=\frac{1}{\rho} \int_{0}^{\nu} x p(x) d x$. Gini index can be then defined as $G=2 \int_{0}^{1}(\nu-L(\nu)) d \nu$ (7). It assumes values between 0 percent (perfect equality) and 100 percent (perfect inequality).

Recently we developed a simple parameter free model for the competition among different economic agents within a conservative model. Results for the wealth distribution has been published (8) and also considering the effect of a small world network (9). Within this model we obtained an exponential Gibbsstyle distribution for the wealth, with a finite lower threshold or poverty line, that is characteristic of models with extremal dynamics. In spite of the fact that this kind of distribution seems to be at stake when compared with Pareto law, the idea of a society that take measures in order to improve the situation of the most impoverished is compatible with the propositions of John Rawls, in his book "A Theory of Justice" (10), directed towards an inventive way of securing equality of opportunity as one of the basic principles of justice. He asserts that no redistribution of resources within... a state can occur unless it benefits the least well-off and this should be the only way to prevent the stronger (or richer) from overpowering the weaker (or poorer). It seems that a 
minimum dynamics, as that proposed in the Bak-Sneppen model (11), when applied to economics, is a way to ensure to the poorest agent a chance to improve its situation.

In the next section we resume the principal assumptions of the model and the principal results of the numerical simulations. The calculation of the Gini index and the comparison with wealth distribution of several countries will be presented in section 3 along with the conclusions

\section{The Conservative Exchange Market Model}

The Conservative Exchange Market Model (CEMM) (8) is a simple macroeconomic model that consists of an one-dimensional lattice with $N$ sites and periodic boundary conditions, where every site represents an economic agent (individuals, industries or countries). To each agent it is assigned some wealthparameter that represents its welfare, like the GNI for countries or salary for individuals. We choose an initial configuration where the wealth is a number between 0 and 1 distributed randomly among agents. The dynamics of the system is supported on the idea that some measure should be taken to modify the situation of the poorest agent. In this context, we model this process by a minimum dynamics (11): at each time step, the poorest agent, i.e., the one with the minimum wealth, will take some action, trying to improve its economic state. Since the outcome of any such measure is uncertain, the minimum suffers a random change in its wealth (8). We assume that whatever wealth is gained (or lost) by the poorest agent it will be at the expense of its neighbors and we assume that it is equally debited (or credited) between its two nearest neighbors on the lattice, making the total wealth constant. So, the CEMM dynamic can be reduced as follows:

- Find out the site with minimum wealth $x_{m i n}$;

- Substitute $w\left(x_{\text {min }}\right)$ to $w_{n e w}$, a new random number between 0 and 1 extracted from an uniform distribution;

- Make the difference, $\Delta=w_{\text {new }}\left(x_{\text {min }}\right)-w\left(x_{\text {min }}\right)$

- Updating the neighbors:

$w\left(x_{\min }-1\right)=w\left(x_{\min }-1\right)-\Delta / 2$

$w\left(x_{\min }+1\right)=w\left(x_{\min }+1\right)-\Delta / 2$

Note that if $\Delta$ is positive (negative) the wealth of the neighbors will be reduced (increased) by $\Delta / 2$.

Numerical simulations on this model showed that, after a relatively long transient, the system arrives at a self organized critical (SOC) state with a stationary wealth distribution (figure 1(8) ) in which almost all agents are beyond a certain threshold. On Fig1 we represent both the cases of local exchanges and 


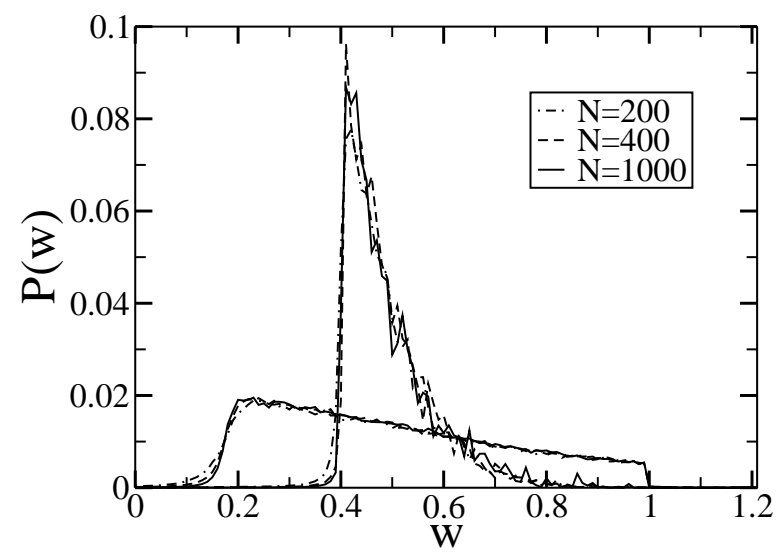

Fig. 1. Distribution wealth $P(w)$ for the model with interactions to nearest neighbors (upper curves) and with global interactions (lower curves). In both cases we have superposed scaled data corresponding to $N=200,400,1000$. The histograms were built using $10^{5}$ consecutive states, after a transient of $10^{6}$ steps has elapsed.

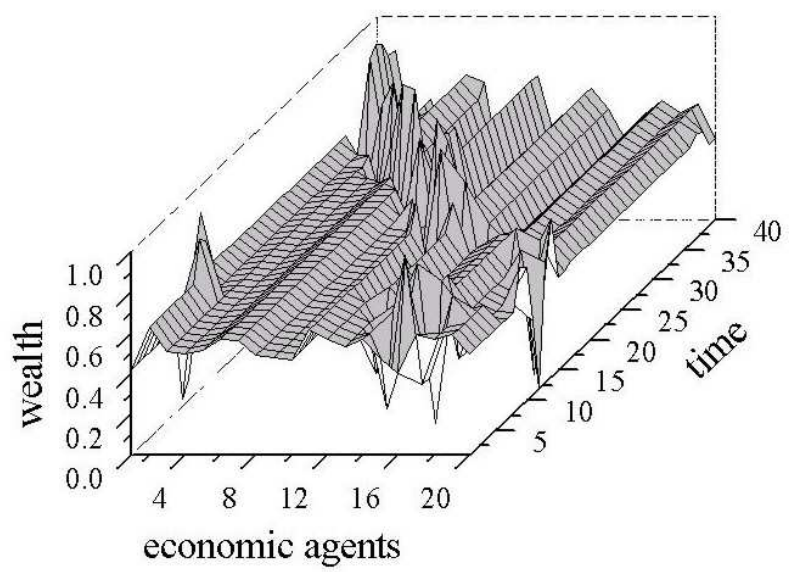

Fig. 2. Snapshot of avalanche propagation for the local case. The sites with maximum wealth are very stable in time.

of global exchanges (mean field solution); in the former case the poverty line is $\eta_{T} \approx 0.4$ and above this threshold the distribution of agents is an exponential $P(w) \approx \exp \left(-w^{2} / 2 \sigma^{2}\right)$, with $\sigma=0.228(9)$, i.e., there are exponentially few rich agents while the mass of them remains in what we call a middle class. In the second case the figure show the globally coupled (mean field) exchanges (lower curves). This corresponds to a situation in which the agents with which the exchange takes place are chosen at random and not based on geographical proximity. This mean field solution exhibits a lower threshold, $\eta_{T} \approx 0.2$, and beyond it also an exponential distribution, $P(w) \approx \exp \left(-w^{2} / 2 \sigma^{2}\right)$, but with a higher value of $\sigma=0.567(9)$.

The temporal evolution, in the SOC state, of the position of the system's minimum and maximum wealth is quite different. While the site of minimum wealth is changing continuously, generating avalanches of all sizes among 


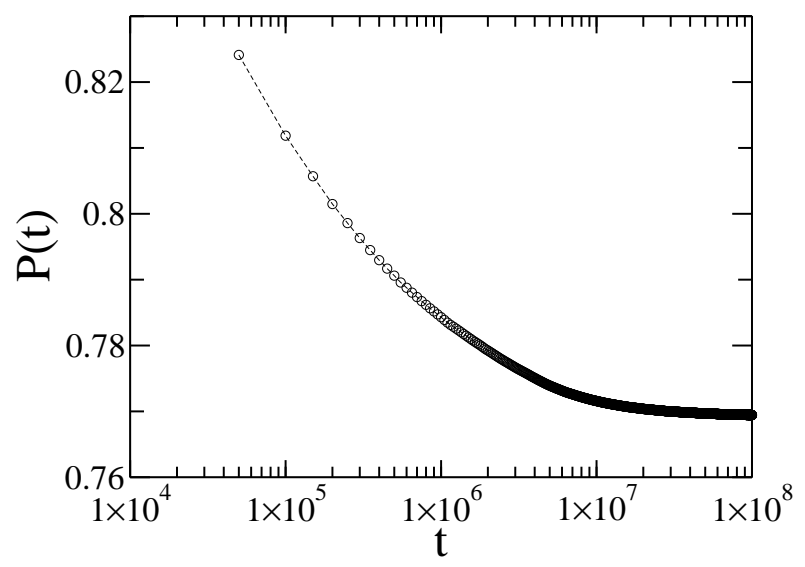

Fig. 3. Probability of an agent become wealthier in a time step for a market with $N=1000$ agents.

neighbors, the wealthiest site is stable over relatively long periods of time (8) as it is represented by the lines in the figure 2. Indeed, even when affected by an avalanche the maximum can frequently recover its status after a short time. Not only the absolute maximum is stable, but also a privileged group, whose wealth is around the same value of the maximum, remains in its prosperous position for quite a while. This group is also privileged by having high wealth values, well above the market average. In the mean-field case the rich sites are also stable, but over shorter periods of time.

We also observed that the probability that the minimum asset agent improve its situation in a time step is relatively high. It is very high at the beginning of the simulation, then it decreases during the transient to finally converge to a finite value, $p \approx 0.77$ as it is shown on figure 3 . We remark that the probability is well above 0.5 , so this minimum dynamic has the effect of improve, in the average, the situation of the poorer agents.

\section{$3 \quad$ Income inequality}

Inequalities are related to social and economic phenomena and is a subject of much debate. The fraction of rich and poor people in a country depends on the inequalities of the income distribution. The standard way to determine the inequality of a wealth distribution is with the help of quintile tables that show the percentage of national income received by equal percentiles of individuals or households ranked by their per capita income levels. For example in a welfare state as Sweden the richest 20 percent of the population receives about 4 times more than the poorest quintile, while in a ruthless capitalistic country like Brazil the richest quintile receives more than 25 times more than the poorest quintile. Table 1 gives the quintile data for a few countries, while full data can be obtained in the World Bank site (12; 13). 


\begin{tabular}{lcccc}
\hline & USA (1997) & Sweden (1992) & Spain (1990) & Brazil (1998) \\
\hline Poorest 20\% & 5.2 & 9.6 & 7.5 & 2.2 \\
\hline Fourth 20\% & 10.5 & 14.5 & 12.6 & 5.4 \\
\hline Third 20\% & 15.6 & 18.1 & 17.0 & 10.1 \\
\hline Second 20\% & 22.4 & 23.2 & 22.6 & 18.3 \\
\hline Richest 20\% & 46.4 & 34.5 & 40.3 & 64.1 \\
\hline Gini index & 40.8 & 25.0 & 32.5 & 60.7 \\
\hline
\end{tabular}

Table 1

Percentage of income in each population quintile for some selected countries (13).

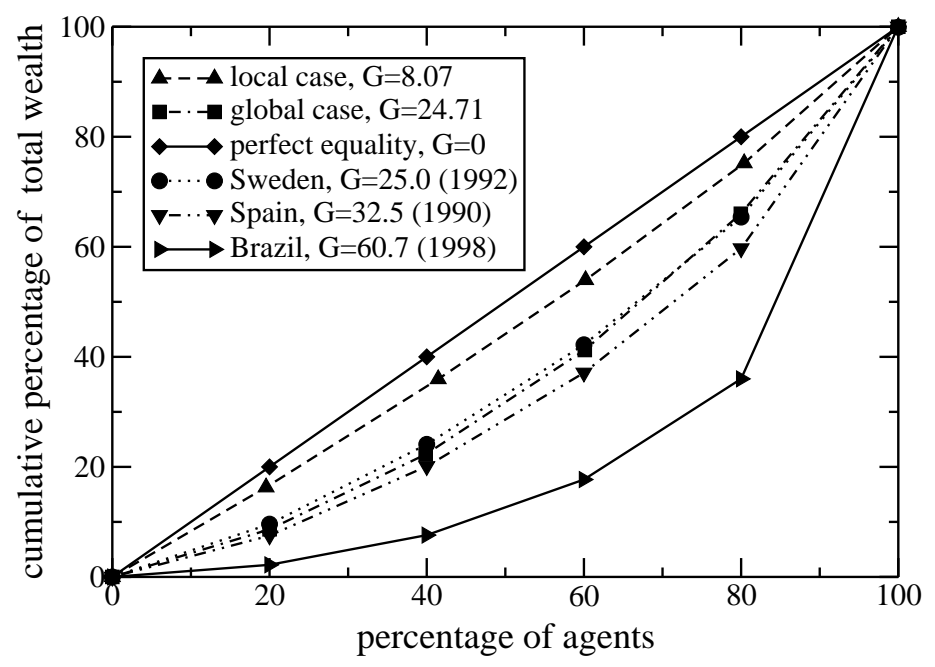

Fig. 4. The Lorenz curves and the Gini index for ideal, local and the global case. Also represented are the World Bank data for Brazil, Sweden and Spain (13).

To measure income inequality in a country and to make a comparison among countries more accurately, economists use Lorenz curves and Gini indexes. A Lorenz curve plots the cumulative percentages of total income against the cumulative percentages of recipients. In Fig 4 we have represented the Lorenz curves for Brazil, Sweden and Spain. For comparison, we have plotted in same figure the Lorenz curve of an hypothetical absolutely equalitarian income distribution that is the straight line with slope equal 1. This figure shows that Brazil's Lorenz curve strongly deviates from the hypothetical line of absolute equality while that for Sweden lies much nearer and that for Spain in the middle of both. This means that of these three countries, Brazil has the highest income inequality and Sweden the lowest. We also constructed Lorenz curves for the local and global cases of CEMM model (figure 4). Clearly we see that the global case have a Lorenz curve very similar to Sweden, while the local case is close to perfect equality. 
The Gini index is even more convenient than a Lorenz curve when the task is to compare income inequality among many countries; it is calculated as the area between a Lorenz curve and the hypothetical line of absolute equality. It assumes values between 0 percent (perfect equality) and 100 percent. Of course, real Gini indexes are always in between. We calculated the Gini index for the local and global cases. For the local case, the Gini index is 8.07, close to perfect equality, while its value is 24.71 for the global one (figure 4 ). This last value is very close to that Sweden (25.0), and also close to values of other European developed countries.

Concluding, the analysis of the Gini index shows that globalization (mean field case) increases the economic inequalities in a conservative economy. Moreover, the poverty line in global case is well bellow that of the local case (figure 1). Another interesting aspect is that the minimum dynamic favors the poorest agent, so promoting wealth redistribution in the sense defined by Rawls (10). Also, from a strictly economic point of view, high inequality means lower exchanges, and so it implies in a reduction of the economic activity.

\section{References}

[1] V. Pareto, Cours d'economie politique, Vol. 2, F. Pichou, Lausanne and Paris, 1897.

[2] J.-P. Bouchaud and M. Potters, Theory of Financial Risks: from statistical physics to risk management, (Cambridge University Press, Cambridge, 2000) (First Edition).

[3] W. Souma, Fractals 9 (2001), 463

[4] V. Dragulescu and V. M. Yakovenko, Eur. Phys. J. B 299(2001), 213

[5] http://www.ibge.gov.br/home/estatistica/populacao/trabalhoerendimento /pnad99/sintese/grafico.shtm

[6] As 500 maiores sociedades anônimas do Brasil, Fundação Getulio Vargas, www.fgv.br/IBRE/CEAE/arq/x500M.xls

[7] C. Gini, Indici di concentrazione e di dispendenza, Biblioteca delli'economista 20 (1922).

[8] S. Pianegonda, J. R. Iglesias, G. Abramson and J. L. Vega, Phys. A 322 (2003), 667

[9] J. R. Iglesias, S. Gonçalves, S. Pianegonda, J.L. Vega and G. Abramson, Physica A 327 (2003), 12

[10] J. Rawls, A Theory of Justice, The Belknap Press of Harvard University Press, Cambridge, MA, 1971.

[11] P. Bak, K. Sneppen, Phys. Rev. Lett. 71 (1993), 4083

[12] http://www.worldbank.org/data/quickreference/quickref.html

[13] http://www.worldbank.org/poverty/data/2_8wdi2002.pdf. 